\title{
A Potential Biodegradable Mg-Y-Ag Implant with Strengthened Antimicrobial Properties in Orthopedic Applications
}

\author{
Yilong Dai ${ }^{1,2,3}{ }^{1}$, Hui Liu ${ }^{2,3}$, Yifu Tang ${ }^{1}$, Xuemei $\mathrm{Xu}^{2,3}$, Haitao Long ${ }^{1}$, Yang Yan ${ }^{2,3}$, \\ Zhenhua Luo ${ }^{1}$, Yu Zhang ${ }^{2,3}$, Kun Yu ${ }^{2,3}$ and Yong Zhu ${ }^{1, *}$ \\ 1 Department of Orthopedics, Xiangya Hospital, Central South University, Changsha 410008, China; \\ daiyilong@live.cn (Y.D.); tangyifu12@hotmail.com (Y.T.); doclong2008@aliyun.com (H.L.); \\ docluozh@yahoo.com (Z.L.) \\ 2 Department of Material Science and Engineering, Yantai Nanshan University, Yantai 265713, China; \\ liuhuirise@csu.edu.cn (H.L.); xuxuemei@csu.edu.cn (X.X.); yanyang1017@gmail.com (Y.Y.); \\ zhangyu2016@csu.edu.cn (Y.Z.); yukun2010@csu.edu.cn (K.Y.) \\ 3 School of Material Science and Engineering, Central South University, Changsha 410083, China \\ * Correspondence: doczhu2003@aliyun.com; Tel.: +86-0731-89753005
}

Received: 16 October 2018; Accepted: 12 November 2018; Published: 14 November 2018

\begin{abstract}
In order to design a potential biodegradable implant, which combines with fine mechanical and antimicrobial properties, $\mathrm{Mg}-4 \mathrm{Y}-1 \mathrm{Ag}$ (mass fraction, \%) alloys were produced by permanent mold casting and then hot extrusion. The microstructure, mechanical behavior, anti-corrosion behavior, and antimicrobial properties of the experimental alloys were comprehensively investigated. The results showed that $\alpha-M g, M_{24} Y_{5}(\varepsilon)$, and $\mathrm{AgMg}_{4}$ phases existed in the Mg-4Y-1Ag. The grain size of $\mathrm{Mg}-4 \mathrm{Y}-1 \mathrm{Ag}$ was greatly refined through hot-extrusion. The as-extruded Mg-4Y-1Ag alloy exhibit an ultimate tensile strength of 202.7 MPa with a good elongation of 33.6\%. The compressive strength of as-extruded Mg-4Y-1Ag was $385 \mathrm{MPa}$, and the strength remained $183 \mathrm{MPa}$ after immersing in PBS solution for four weeks. The as-extruded alloy had better corrosion resistance than as-cast alloy and as-extruded pure magnesium in PBS solution, for the reason of refined grain and the formation of $\mathrm{Y}_{2} \mathrm{O}_{3}$ film on the surface of Mg-4Y-1Ag alloy. Furthermore, the as-extruded Mg-4Y-1Ag alloys were superior to Ti6Al4V (TC4) and as-extruded pure magnesium in antimicrobial property for released $\mathrm{Ag}^{+}$ion. Obvious inhibition halo was observed in the LB agar plate adding with as-extruded Mg-4Y-1Ag alloys. Also as-extruded Mg-4Y-1Ag alloys showed no cytotoxicity by co-culturing with L929 using the MTT method.
\end{abstract}

Keywords: biomaterials; mechanical strength; corrosion; antimicrobial; cytotoxicity

\section{Introduction}

Magnesium and its alloys have been investigated as biodegradable metallic materials in bone implants over the past years due to their inherent biocompatibility, biodegradability, and their excellent mechanical properties close to the natural bone [1,2]. Mg and its alloys may be a good substitution for permanent materials such as stainless steels, Ti-based alloys, polymers, or bioceramics which do not require a second surgery [3].

However, the clinical application of magnesium alloys is limited by their rapid corrosion speed [4,5], which is almost an intrinsic response of magnesium to a chloride containing solution [6,7], like the human body fluid. Meanwhile, a lot of hydrogen gas will be released during the process of rapid corrosion. If the evolution is too rapid to be absorbed, a balloon effect will take place and an alkaline $\mathrm{pH}$ shift will happen in the vicinity of the corroded surface [8]. Moreover, the biodegradable 
implants made of magnesium alloys can't keep mechanical integrity before the patients recovered due to the rapid degradation rate [9]. In addition to consider the corrosion of magnesium alloy without stress, magnesium alloy implants possess adequate resistance to cracking/fracture under stress corrosion cracking (SCC), before the implant can be put to actual use [10]. The AZ91D alloy is found to be susceptible to SCC in the physiological environment. A substantial decrease in the mechanical integrity of AZ91D is observed in investigations using the SSRT testing in m-SBF solution. Therefore, controlling the corrosion rate of magnesium in body fluid is an important issue in the development of magnesium implants and one of the most effective measures is alloying treatment [11].

Silver (Ag) is well known for its strong antimicrobial property for thousands of years. Ag ions released from the degradation of $\mathrm{Mg}-\mathrm{Ag}$ alloys could kill bacteria during bone healing [12]. Additionally, the addition of Ag was reported to enhance the corrosion resistance of $\mathrm{Mg}$ alloys due to its noble nature and contribution to the formation of oxidation films [13]. $\mathrm{Mg} 2 \mathrm{Ag}$ (wt \%) showed good corrosion resistance and promising antibacterial activity among $\mathrm{Mg}, \mathrm{Mg} 2 \mathrm{Ag}, \mathrm{Mg} 4 \mathrm{Ag}$, and $\mathrm{Mg} 6 \mathrm{Ag}$. It was also reported that RE element (Y) can promote the formation of protective surface films which then strengthen the anti-corrosion ability of magnesium alloys [14]. According to the study of He et al. [15], when the yttrium weight content was above $0.36 \%$ in Magnesium alloy, $\mathrm{Y}_{2} \mathrm{O}_{3}$ protective surface film would form on exposure to Hank's solution. Moreover, the $\mathrm{Y}_{2} \mathrm{O}_{3}$ film promoted the anti-corrosion ability by significantly decreasing the cathodic current density. Therefore, we developed the Mg-4Y-1Ag alloy for promoting both antimicrobial and anti-corrosion properties in this research.

Except alloying treatment, thermomechanical processes are also an important method to improve the mechanical properties and corrosion resistance of magnesium alloys. Nayak et al. [16] found that Grain size got refined significantly with increasing amount of deformation. The $\mathrm{Mg}-3 \mathrm{Zn}$ (wt \%) experiences a marked improvement in hardness, yield strength, ultimate tensile strength, strain and toughness with finer grain size through hot-rolled. $\mathrm{Mg}-\mathrm{Zn}$ alloys and $\mathrm{Mg}-\mathrm{Zn}-\beta-\mathrm{Ca}_{3}\left(\mathrm{PO}_{4}\right)_{2}$ composites shows fine comprehensive properties in as-extruded state [17]. As hot-extrusion could densify magnesium alloy better than hot-rolling for its severe deformation, hot-extrusion is selected as further method to process Mg-Y-Ag alloy.

On one hand, Mg-Re alloy shows good corrosion resistance and mechanical properties among magnesium alloys, on the other hand, $\mathrm{Mg}-\mathrm{Ag}$ alloy proves to be good antimicrobial biodegradable implant. We hope to design Mg-Y-Ag alloy to occupy both corrosion resistance, mechanical properties, and antimicrobial properties for biodegradable implants. Moreover, Marián Viček [18] compared in vitro corrosion of $\mathrm{Mg}-4 \mathrm{Y}$ and $\mathrm{Mg}-4 \mathrm{Y}-1 \mathrm{Ag}$, and made a conclusion that the addition of $1 \mathrm{wt} \% \mathrm{Ag}$ to the Mg-4Y alloy has practically no effect on the in vitro corrosion rate in the solution-treated condition. In the present work, Mg-4\% $-1 \% \mathrm{Ag}$ (wt \%) alloys were prepared and hot-extruded to study the microstructure, mechanical, corrosion, and antimicrobial properties compared to pure magnesium and Ti6Al4V (TC4). Meanwhile, its cytotoxicity was evaluated by the MTT method.

\section{Materials and Methods}

\subsection{Sample Preparation}

The Mg-4\%Y-1\%Ag (wt \%) alloy was melted with high purity Mg (>99.9\%), Ag (>99.99\%) and Mg-20\%Y (wt \%) master alloy. The actual chemical composition of Mg-4\%Y-1\%Ag alloy was determined by inductively coupled plasma atomic emission spectroscopy (ICP-AES), which was Mg-3.73\%Y-0.85\%Ag. The as-cast Mg-4Y-1Ag (C-Mg-4Y-1Ag) ingot with a size of $\varphi 60 \mathrm{~mm} \times 200 \mathrm{~mm}$ was homogenized at $400{ }^{\circ} \mathrm{C}$ for $24 \mathrm{~h}$ and hot-extruded at $350{ }^{\circ} \mathrm{C}$ to a plate with extrusion ratio of 12:1. The experimental samples of as-extruded Mg-4Y-1Ag (Ex-Mg-4Y-1Ag) and as-extruded pure magnesium (Ex-Mg) were both obtained from the extruded plates, while samples of the as-cast alloys were cut from the casted ingot. Disk samples $10 \mathrm{~mm} \times 10 \mathrm{~mm} \times 4 \mathrm{~mm}$ in size were prepared for the immersion test and electrochemical measurements. The specimens were grounded with 1000 grid $\mathrm{SiC}$ 
sandpaper and diamond polishing, then etched with etchant solution. The etchant used for optical microstructure observation was composed of $5 \mathrm{~mL}$ nitric acid and $95 \mathrm{~mL}$ absolute ethyl alcohol.

\subsection{Mechanical and Metallurgical Testing}

Mechanical testing samples were cut from the ingot and the extruded plate. The tensile samples ( $40 \mathrm{~mm}$ in gauge length and $6 \mathrm{~mm}$ in diameter) and compressive samples $(10 \mathrm{~mm}$ in gauge length and $6 \mathrm{~mm}$ in diameter) were machined from the extruded plates along the extrusion direction. The tensile tests and compressive tests were conducted at a crosshead speed of $2 \mathrm{~mm} / \mathrm{min}$ at room temperature in air using a MTS type machine (MTS 810, MTS Systems Coporation, Minnesota, MI, USA). The elastic modulus, yield stress, and ultimate strength of tensile tests were calculated by the software attached with MTS machine. At least three samples were tested for each condition. The microstructures of the experimental Mg-4Y-1Ag specimens were characterized using optical microscope (OM, Leica DMi8, Solms, Germany) and scanning electron microscope (SEM, FEI Quanta 200, Hillsboro, OR, USA). Presence of each phases were identified with a BRUKER D8 X-ray diffraction (XRD) employing $\mathrm{CuK}_{\alpha}$ radiation $(\lambda=1.5406 \AA)$. The scan range was performed continuously from $10 \mathrm{deg}$ to $80 \mathrm{deg}$ at a speed of $2 \mathrm{deg} / \mathrm{min}$.

\subsection{In Vitro Corrosion Testing}

Immersion testing was performed in phosphate buffer saline (PBS) at $37 \pm 0.2{ }^{\circ} \mathrm{C}$ for two weeks to evaluate the in vitro corrosion properties of experimental alloys. The $\mathrm{pH}$ value of PBS was recorded during the immersion. In immersion test, the PBS solution didn't change to make convenient to measure the $\mathrm{pH}$ value of the solution. The $\mathrm{H}_{2}$ gas evolution testing was also carried out in PBS at $37 \pm 0.2{ }^{\circ} \mathrm{C}$ for 2 weeks to evaluate the volume of evolved $\mathrm{H}_{2}$ gas and corrosion rate of $\mathrm{C}-\mathrm{Mg}-4 \mathrm{Y}-1 \mathrm{Ag}$, Ex-Mg-4Y-1Ag and Ex-Mg. The PBS solution in hydrogen evolution tests was renewed every $24 \mathrm{~h}$ in order to maintain a relatively stable fluid environment [19]. The working area of specimens used for electrochemical testing was $1 \mathrm{~cm}^{2}$, which was ground with 400, 600, 800, and 1000 grid sandpaper, respectively. The electrochemical testing was performed at $37 \pm 0.2^{\circ} \mathrm{C}$ by a three-electrode device (the specimens as working electrode, saturated calomel as reference electrode and platinum foil as the counter electrode) with a CHI660C potentiostat/galvanostat system (CHI Company, Austin, TX, USA). Tafel extrapolation approach was adopted to measure the corrosion current density (Icorr), corrosion potential (Ecorr) and polarization resistant (Rp) of samples. Electrochemical impedance spectroscopy (EIS) was examined with a perturbation amplitude of $10 \mathrm{mV}$. The scanning frequency of EIS ranged from 10,0000 to $0.01 \mathrm{~Hz}$. ZsimDemo 3.30d software (EChem Software, Michigan, MI, USA) was used to fit and analyze the EIS results.

\subsection{Antibacterial and Ion Concentration Testing}

The samples, including Ex-Mg-4Y-1Ag, Ex-Mg and TC4 (Ti6AL4V) were denoted as S1, S2, Z, and S3 respectively. Staphylococcus aureus (ATCC 25923) was selected to evaluate the antibacterial properties of the specimens. Three specimens and a blank control group, with $10 \mathrm{~mL}$ of the solution which contained bacteria medium, the concentration of which was $10^{6} \mathrm{CFU}$ (colony-forming units) $\cdot \mathrm{mL}^{-1}$, were incubated on a 6-well culture plate at room temperature for $24 \mathrm{~h}$. The antibacterial ratio was calculated as the equation: Antibacterial rate $(\%)=(\mathrm{CFU}$ of control group $-\mathrm{CFU}$ of testing groups)/CFU of control group $\times 100 \%$. The antibacterial abilities of the Ex-Mg-4Y-1Ag was also assessed by the inhibition halo testing with $\mathrm{S}$. aureus strain. Then, $10^{5} \mathrm{CFU} / \mathrm{mL}$ bacterial suspension was made up and evenly distributed on a LB agar plate, all the specimens were put in touch with the agar in the middle and cultured at $37^{\circ} \mathrm{C}$ for $24 \mathrm{~h}$. Three groups of specimens and $10 \mathrm{~mL}$ of suspension $\left(10^{6} \mathrm{CFU} \cdot \mathrm{mL}^{-1}\right)$ were incubated on agar plate at $37^{\circ} \mathrm{C}$ for 2 and $4 \mathrm{~h}$ respectively. The suspension with samples were cultured for 2 and $4 \mathrm{~h}$, respectively, and then were in contact with BacLight Live/Dead which utilizes Syto9 and propidium iodide (PI) reagent mixture. Then, the specimens were washed third times by PBS solution. Finally, the fluorescence microscopy (NIKON, Tokyo, Japan) was used to 
evaluate the quantity of bacteria. The ion concentration variation of $100 \mathrm{~mL}$ medium was monitored by an inductively coupled plasma optical emission spectrometry (ICP-OES). $10 \mathrm{~mL}$ of $100 \mathrm{~mL}$ culture medium was taken out to test and $10 \mathrm{~mL}$ fresh culture medium would be added into the remaining medium every week.

\subsection{Cell Toxicity Experiment}

According to the ISO 10993-2009 standard, mouse fibroblast L929 cells (ATCC CCL-1) were cultured with extracting fluid $(10 \%, 50 \%$, and 100\%), the Dulbecco's modified Eagle's medium (DMEM) as negative control and the DMEM containing $0.5 \%$ phenol as positive control in different time points respectively. The cell toxicity of material extract was measured by 3-(4,5-dimethylthiazol-2-yl)-2,5-diphenyltetrazolium bromide (MTT) method, and the relative growth rate (RGR) was calculated according to the OD values to evaluate cell toxicity based on ISO 10993-2009 standard. The morphology of L 929 cells were observed by optical microscope at $72 \mathrm{~h}$ of incubation.

\subsection{Statistical Analysis}

The antibacterial tests and cell toxicity tests were performed at least three times, and the results were expressed as mean $\pm \mathrm{SD}$, and statistical analysis between different groups was performed with one-way ANOVA using the SPSS 17.0 software (IBM, Armonk, NY, USA). Differences were considered significant when ${ }^{*} p<0.05$.

\section{Results}

\subsection{Microstructure of $M g-4 Y-1 A g$ Alloys}

The typical micrographs of the as-cast and as-extruded Mg-4Y-1Ag alloys taken by optical microscopy are shown in Figure 1a,b. The as-cast alloys had fine grain size of 100-200 $\mu \mathrm{m}$. The secondary dendrites are observed in the grains. Some eutectic phases form during the solidification along the grain boundaries, while the grain size of as-extruded alloys is $10-20 \mu \mathrm{m}$. The grain size was well refined by hot extrusion which eliminate the second dendrites in grain at the same time. Representative secondary electron image of the as-extruded alloys morphology is illustrated in Figure $1 c, d$, and the white second phases can also be seen in the SEM image. Combined with XRD patterns (Figure 1e) and EDS, the white second phases along the extrusion direction are $\mathrm{Mg}_{24} \mathrm{Y}_{5}(\varepsilon)$ and $\mathrm{AgMg}_{4}$, the quantity and size of which decrease through hot-extrusion. The $\varepsilon$ phases were the bright white spots in position A. However, the dark white spots in position B were the mixtures of $\varepsilon$ and $\mathrm{AgMg}_{4}$ phases. Moreover, the hot-extrusion does not change the phases of $\mathrm{Mg}-4 \mathrm{Y}-1 \mathrm{Ag}$ alloys, only change the orientation of grains due to the variations of X-ray diffraction peak intensity in (100) and (002) lattice plane of Mg matrix. 

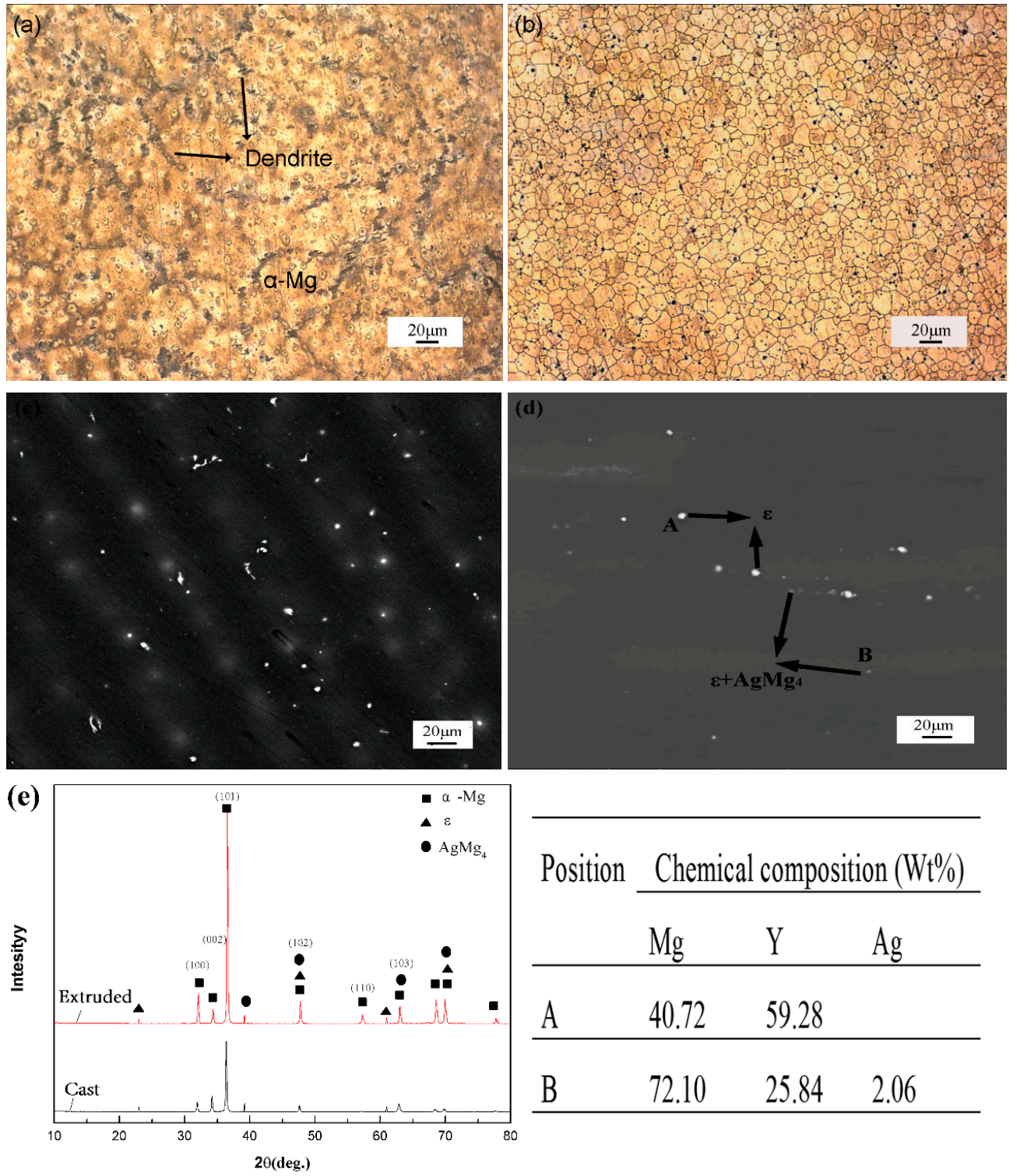

\begin{tabular}{llll} 
A & 40.72 & 59.28 & \\
\hline B & 72.10 & 25.84 & 2.06 \\
\hline
\end{tabular}

Figure 1. The microstructure and XRD patterns of Mg-4Y-1Ag alloys: (a) optical image of as-cast alloys; (b) optical image of as-extruded alloys; (c) SEM image of as-cast alloy; (d) SEM image of as-extruded alloys; and (e) XRD patterns and EDX analysis of Mg-4Y-1Ag alloys.

\subsection{Mechanical Property of Mg-4Y-1Ag Alloys}

Table 1 summarizes the mechanical properties of the $\mathrm{Mg}-4 \mathrm{Y}-1 \mathrm{Ag}$ alloys compared with the equivalent properties of natural bone [3], Ex-Mg and TC4. The elastic modulus of Mg-4Y-1Ag and Ex-Mg is close to that of natural bone, while the elastic modulus of TC4 is much higher than that of natural bone. Therefore, it can avoid the stress shielding effect induced by a serious mismatch between the modulus of natural bone and other metal implants. The tensile testing results, as shown in Figure 2a, indicate that the improvement in yield stress, ultimate tensile stress, and elongation of Ex-Mg-4Y-1Ag compared with C-Mg-4Y-1Ag. Since the grain size of Mg-4Y-1Ag is refined by hot-extrusion, the strength increases with the decrease of the grain size according to the Hall-Petch equation. Moreover, Ex-Mg-4Y-1Ag alloys have better mechanical strength and elongation with the addition of $\mathrm{Y}$ and Ag element than Ex-Mg. The addition of Y [20] improves the strengths and the addition of Ag [12] improves the elongation in Magnesium binary alloys. The Ex-Mg-4Y-1Ag showed good ultimate tensile strength (UTS) and elongation of $211 \mathrm{MPa}$ and $40.4 \%$, which meet specific mechanical requirements that the strength should be higher than $200 \mathrm{MPa}$ and the elongation greater 
than $10 \%$ for biomaterials [21]. Figure $2 \mathrm{~b}$ shows the fracture morphology of Ex-Mg-4Y-1Ag. Many deep dimples distributed in the facture morphology which indicate that Ex-Mg-4Y-1Ag alloy has good plasticity. It has good elongation of $40.4 \%$. Figure $2 \mathrm{c}$,d show the stress-strain compressive curves of Ex-Mg and Ex-Mg-4Y-1Ag immersed in PBS at $37 \pm 0.2^{\circ} \mathrm{C}$ after $0,1,2$, and 4 weeks. It can be seen that the compressive strengths of Ex-Mg-4Y-1Ag and Ex-Mg decrease with the increase of immersion time. Figure 2e shows the ultimate compressive strength-week graph of Ex-Mg and Ex-Mg-4Y-1Ag. The initial compressive strengths of Ex-Mg-4Y-1Ag and Ex-Mg are $385 \mathrm{MPa}$ and $253 \mathrm{MPa}$ before immersion. The Ex-Mg-4Y-1Ag alloy lost much compressive strength which reduced to $265 \mathrm{MPa}$ in one week of immersion. After four weeks of immersion, the corrosion speed of Ex-Mg-4Y-1Ag was slowed down by the formation of corrosion product layer, the compressive strength of Ex-Mg-4Y-1Ag decreases to $183 \mathrm{MPa}$, which is still larger than that of Ex-Mg. Figure $2 \mathrm{f}$ shows a similar phenomenon that the elastic modulus of both Ex-Mg and Ex-Mg-4Y-1Ag decreased with the increase of immersion time.
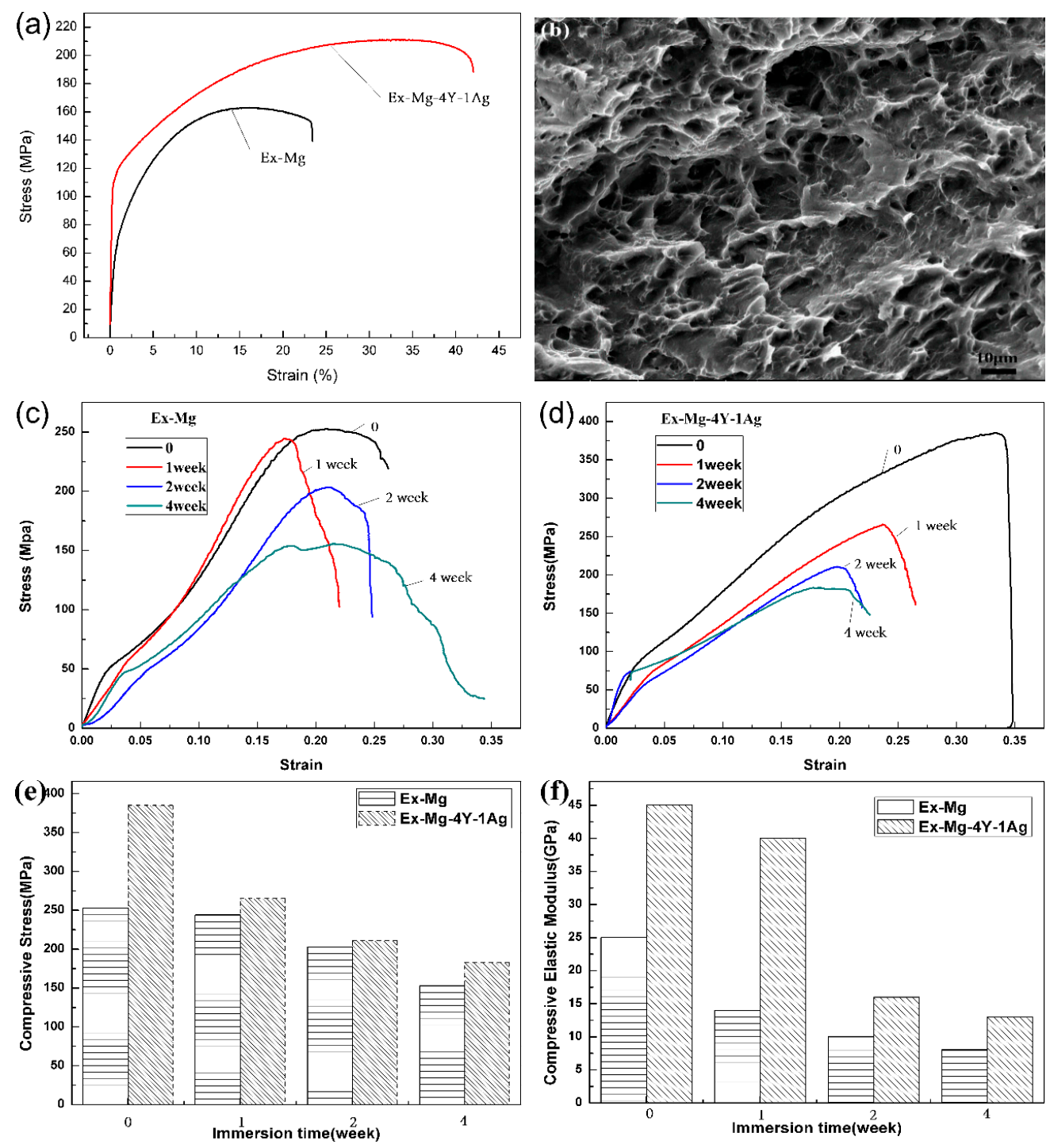

Figure 2. (a) Stress-strain tensile curves of Ex-Mg and Ex-Mg-4Y-1Ag; (b) fracture morphology of Ex-Mg-4Y-1Ag; Stress-strain compressive curves of samples after different immersion time: (c) Ex-Mg-4Y-1Ag, (d) Ex-Mg, (e) ultimate compressive strength-week graph of Ex-Mg and Ex-Mg-4Y-1Ag; and (f) compressive elastic modulus-week graph of Ex-Mg and Ex-Mg-4Y-1Ag. 
Table 1. Mechanical properties of different metal samples.

\begin{tabular}{ccccc}
\hline Samples & Elastic Modulus/GPa & Yield Stress/MPa & Ultimate Tensile Stress/MPa & Elongation/\% \\
\hline C-Mg-4Y-1Ag & 40 & 67 & 146 & 3.8 \\
Ex-Mg-4Y-1Ag & 37 & 102 & 211 & 40.4 \\
Ex-Mg & 21 & 83 & 163 & 9.3 \\
TC4 & 112 & 891 & 986 & 13.0 \\
Natural Bone & $3-20$ & - & $130-180$ & $1.07-2.10$ \\
\hline
\end{tabular}

\subsection{In Vitro Corrosion Test}

Figure 3 compares corrosion properties of C-Mg-4Y-1Ag, Ex-Mg-4Y-1Ag and Ex-Mg in PBS at a temperature of $37 \pm 0.2{ }^{\circ} \mathrm{C}$. The degradation rate of Ex-Mg- $4 \mathrm{Y}-1 \mathrm{Ag}$ is significantly lower than that of the $\mathrm{C}-\mathrm{Mg}-4 \mathrm{Y}-1 \mathrm{Ag}$, while the $\mathrm{pH}$ value, the volume of evolved hydrogen e of Ex-Mg-4Y-1Ag is close to Ex-Mg (Figure 3a,b). Figure 3c shows the potentiodynamic polarization curves of C-Mg-4Y-1Ag, Ex-Mg-4Y-1Ag, and Ex-Mg. Electrochemical parameters were summarized from Figure $3 \mathrm{c}$ by Tafel extrapolation method in Table 2, which indicates that the Ecorr $(-1.791 \mathrm{~V})$ of Ex-Mg is much lower than the Ex-Mg-4Y-1Ag $(-1.464 \mathrm{~V})$ and C-Mg-4Y-1Ag $(-1.537 \mathrm{~V})$, which indicates the addition of Ag and Y promote the Ecor of magnesium. The Jcorr $\left(5.38 \times 10^{-6} \mathrm{~A}\right)$ of the Ex-Mg-4Y-1Ag is much lower than that $\left(1.32 \times 10^{-5} \mathrm{~A}\right)$ of as-cast alloys, while it has the same magnitude order as that $\left(4.92 \times 10^{-6} \mathrm{~A}\right)$ of Ex-Mg, which suggests that hot extrusion can slow down the corrosion rate of Mg-4Y-1Ag alloys.
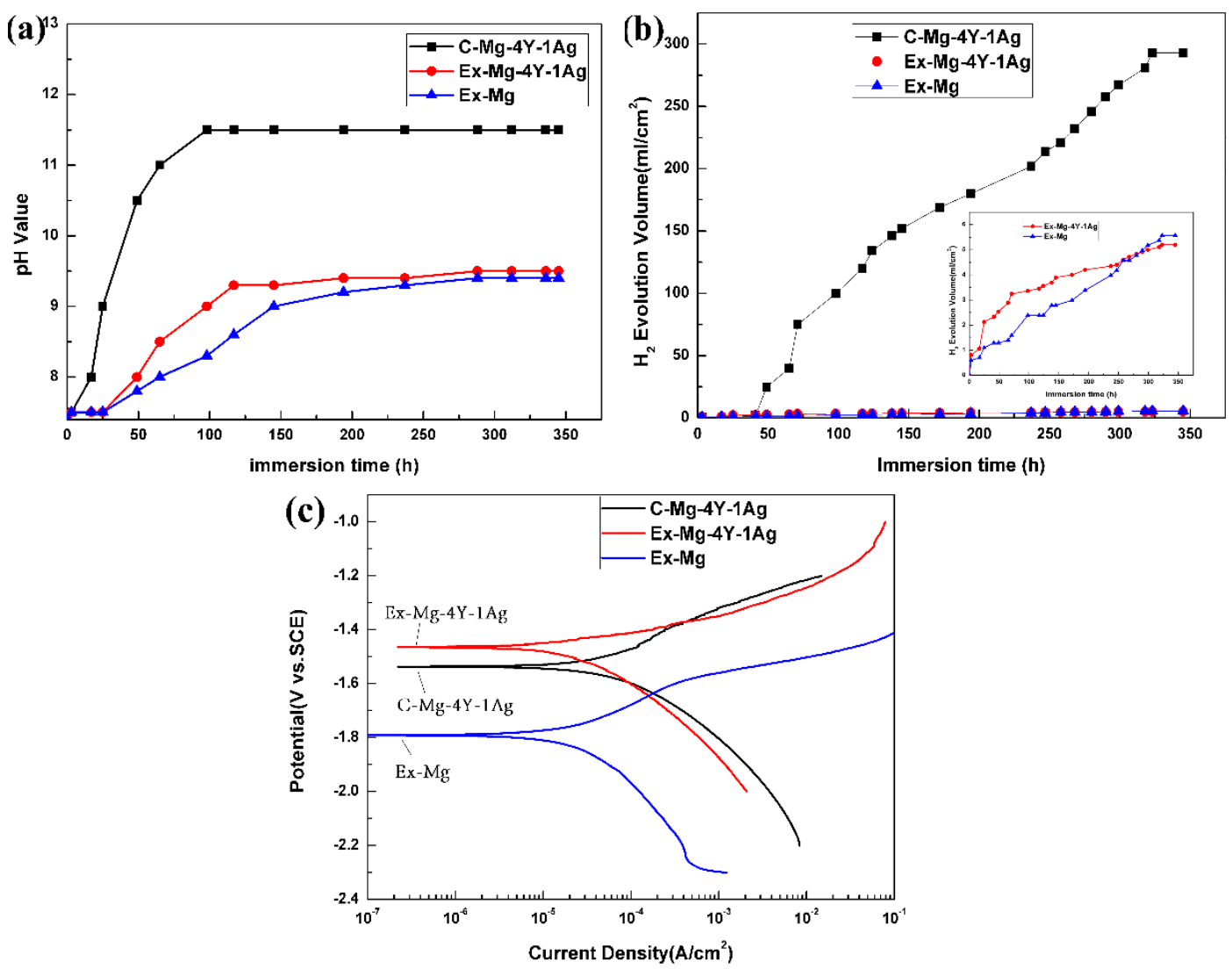

Figure 3. Graphs charting the dynamic degradation of the C-Mg-4Y-1Ag, Ex-Mg-4Y-1Ag and Ex-Mg samples in PBS as a function of time: (a) pH value of PBS; (b) volume of evolved hydrogen; and (c) potentiodynamic polarization curves. 
Table 2. Electrochemical parameters of C-Mg-4Y-1Ag, Ex-Mg-4Y-1Ag, and Ex-Mg.

\begin{tabular}{cccc}
\hline Samples & $\mathbf{E}_{\text {corr }} / \mathbf{V}$ & $\mathbf{I}_{\text {corr }} / \boldsymbol{\mu A}$ & $\mathbf{R}_{\mathbf{p}} / \mathbf{K} \boldsymbol{\Omega}$ \\
\hline C-Mg-4Y-1Ag & -1.537 & 13.2 & 0.732 \\
Ex-Mg-4Y-1Ag & -1.464 & 5.38 & 1.521 \\
Ex-Mg & -1.791 & 4.92 & 1.922 \\
\hline
\end{tabular}

Figure 4 illustrates the surface morphologies of the Ex-Mg and Ex-Mg-4Y-1Ag alloy exposed to PBS solution at $37^{\circ} \mathrm{C}$ for different degradation times. Both Ex-Mg and Ex-Mg-4Y-1Ag alloy specimens experienced pitting corrosion and were covered with corrosion products at the beginning of immersion (24 h), as shown in Figure 4a,b. It can be found that the holes of pitting corrosion in Ex-Mg-4Y-1Ag alloy are larger in quantity than those in Ex-Mg, which means that degradation of Ex-Mg in PBS solution was slower than that of Ex-Mg-4Y-1Ag alloy for the reason of galvanic corrosion caused by the second phases in alloy. The EDS results reveal that the surface corrosion products on Ex-Mg and Ex-Mg-4Y-1Ag alloy samples after immersing in the PBS solution for $24 \mathrm{~h}$ were almost the same, which were rich in $\mathrm{O}, \mathrm{Mg}, \mathrm{P}$ and $\mathrm{Ca}$, as shown in Figure 4. The $\mathrm{P}$ and $\mathrm{Ca}$ components both originate from the PBS solution [22], so the main corrosion product of Ex-Mg and Ex-Mg-4Y-1Ag alloy might be $\mathrm{Mg}(\mathrm{OH})_{2}$ at the beginning of immersion. After $120 \mathrm{~h}$ of immersion time, the surfaces of the entire Ex-Mg and Ex-Mg-4Y-1Ag alloy were found to be covered by the corrosion product layers. The surface morphology of Ex-Mg differed from that of Ex-Mg-4Y-1Ag after soaking in PBS for $120 \mathrm{~h}$, some wide cracks appeared on the entire surfaces of Ex-Mg. The EDS results showed that the element component of zone $\mathrm{C}$ was similar with zone $\mathrm{A}$ and zone $\mathrm{B}$, which means that the main corrosion product of Ex-Mg for $120 \mathrm{~h}$ of immersion was still $\mathrm{Mg}(\mathrm{OH})_{2}$, while a dense corrosion product layer was coated on the surface of Ex-Mg-4Y-1Ag alloy, which might be the mixture of $\mathrm{Mg}(\mathrm{OH})_{2}$ and $\mathrm{Y}_{2} \mathrm{O}_{3}$, because plenty of $\mathrm{Y}$ was detected in zone D (Figure 4D).

EIS plots and the equivalent circuits of Ex-Mg and Ex-Mg-4Y-1Ag in PBS solution are displayed in Figure 5 for further analyzing the corrosion mechanism of the studied alloys. The EIS plots for Ex-Mg and Ex-Mg-4Y-1Ag samples show similar shapes, so their electrochemical mechanism can be explained by the same equivalent circuit. ZSimDemo 3.30d software is adopted to obtain fitted values of impedance parameters. The fitted results are listed in Table 3. The equivalent circuit created from the EIS spectra is presented in Figure 5. The curves of Ex-Mg and Ex-Mg-4Y-1Ag consisted of one high frequency capacitance loop and one low frequency capacitance loop, which was in accord with the [18].

Table 3. Electrochemical parameters of $\mathrm{Mg}$ and Ex-Mg-4Y-1Ag alloy from the electrochemical impedance spectroscopy.

\begin{tabular}{|c|c|c|c|c|c|c|c|}
\hline \multirow{2}{*}{ Samples } & $\mathbf{R}_{\mathrm{s}}$ & $Y_{1}$ & \multirow{2}{*}{$\mathbf{n}_{1}$} & $\mathbf{R}_{\mathrm{t}}$ & $Y_{2}$ & \multirow{2}{*}{$\mathbf{n}_{2}$} & $\mathbf{R}_{\mathrm{f}}$ \\
\hline & $\left(\Omega \cdot \mathrm{cm}^{2}\right)$ & $\left(\mu \Omega^{-1} \cdot \mathrm{cm}^{-2} \cdot \mathrm{s}^{-1}\right)$ & & $\left(\Omega \cdot \mathrm{cm}^{2}\right)$ & $\left(\mu \Omega^{-1} \cdot \mathrm{cm}^{-2} \cdot \mathrm{s}^{-1}\right)$ & & $\left(\Omega \cdot \mathrm{cm}^{2}\right)$ \\
\hline Ex-Mg & 1.830 & 13.47 & 0.9499 & 665.0 & 0.9342 & 1 & 2.404 \\
\hline Ex-Mg-4Y-1Ag & 1.803 & 26.32 & 0.9524 & 430.9 & 45.42 & 0.6483 & 521.6 \\
\hline
\end{tabular}



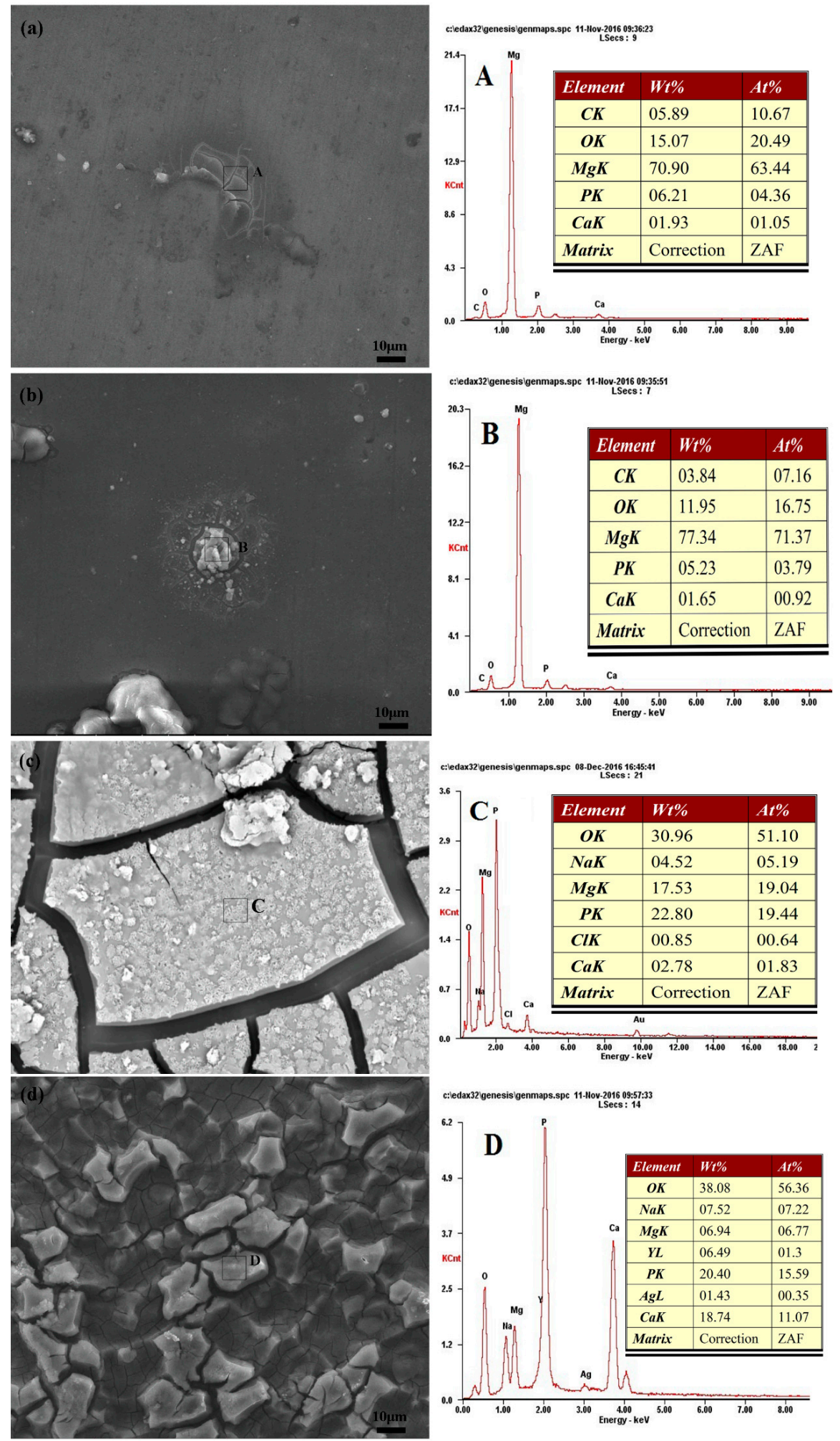

Figure 4. Surface morphologies and EDS analysis of the Ex-Mg and Ex-Mg-4Y-1Ag alloy exposed to PBS solution at $37^{\circ} \mathrm{C}$ for different degradation times: (a) Ex-Mg at $24 \mathrm{~h}$; (b) Ex-Mg-4Y-1Ag at 24 h; (c) Ex-Mg at 120 h; and (d) Ex-Mg-4Y-1Ag at 120 h. EDS in Zone (A) for Figure 4a; Zone (B) for Figure 4b; Zone (C) for Figure 4c; and Zone (D) for Figure 4d. 


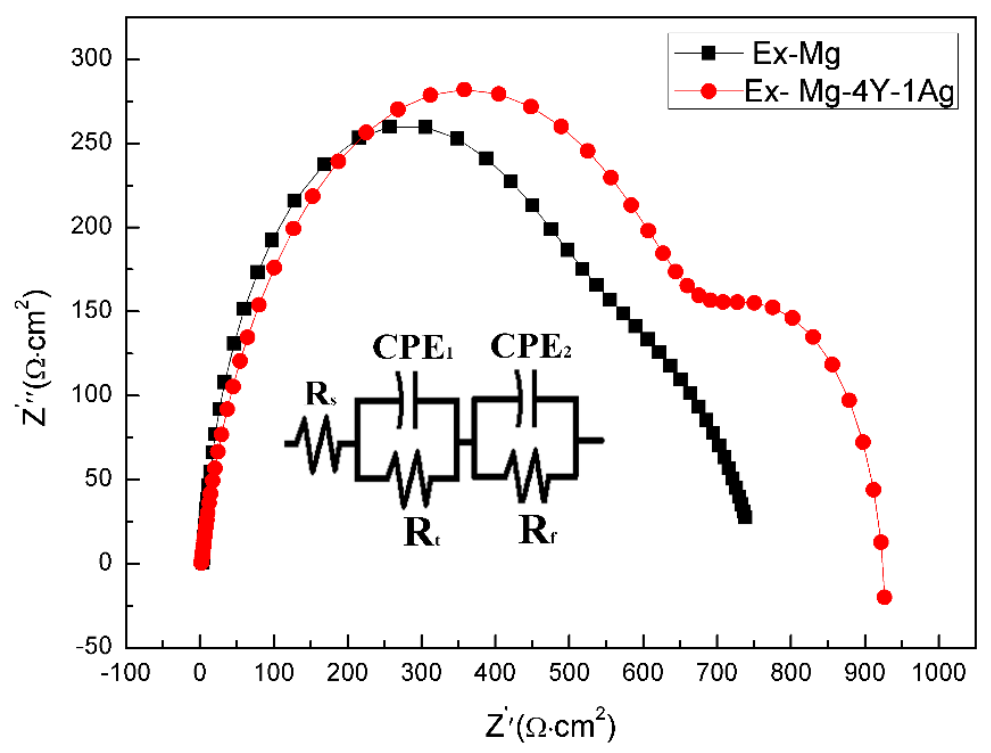

Figure 5. The EIS plots and the equivalent circuit of $\mathrm{Mg}$ and Ex-Mg-4Y-1Ag.

\subsection{Antibacterial Test}

Figure $6 \mathrm{a}$ indicates that in $6 \mathrm{~h}$, group S1 has lesser CFUs than any other groups. The antibacterial rate $(\%)=(\mathrm{CFU}$ of control group - CFU of testing groups) $/ \mathrm{CFU}$ of control group $\times 100 \%$. While co-cultured with the bacteria, the CFU in the Ti group was not less than the control group, so after subtracting CFU values in the control group, the antibacterial rate value may be small, or even a minus. Considering the average Ra value in Ti group is low, the error would be larger. Then the CFUs of all groups increased at $24 \mathrm{~h}$; while group S1 had a very thin colony, the other plates were full of it. As shown in Figure 6b, the antibacterial rate (Ra) values of Ex-Mg-4Y-1Ag, Ex-Mg and TC4, increased from $65.29 \%, 42.55 \%, 28.74 \%$ at $6 \mathrm{~h}$, to $78.98 \%, 55.11 \%, 2.5 \%$ at $12 \mathrm{~h}$, then $92.93 \%, 65.37 \%$, and $23.84 \%$ at $24 \mathrm{~h}$, respectively, indicating that Ex-Mg-4Y-1Ag can inhibit bacteria proliferation, had the strongest antibacterial activity. Figure $6 c$ shows that samples were able to create a clear and significant zone closing to the sample's surface. This behavior is due to the diffusion of $\mathrm{Ag}^{+}$ions. In the area close to the sample the $\mathrm{Ag}^{+}$concentration was high and no bacterial growth was observed, while in the areas further from the sample the silver concentration gradually decreased and some bacterial colonies began to survive.

The fluorescence microscopy images are shown in Figure 7. As the culture duration was prolonged from $2 \mathrm{~h}$ to $4 \mathrm{~h}$, the green fluorescent intensity which represented living bacterial colonies on $\mathrm{Mg}$ and TC4 alloy increased and the red were hardly observed on them, conversely, more red spots were distributed on Ex-Mg-4Y-1Ag, illustrating that the Ex-Mg-4Y-1Ag samples possess better antibacterial activity. According to the counting by Image-Pro, the mean amounts of bacteria are 931.4, 1766, 2413.8 at $2 \mathrm{~h}$ and 999, 2176.4, 2505 at $4 \mathrm{~h}$, respectively, also indicating that the Ex-Mg-4Y-1Ag possess better antibacterial activity among three groups of specimens. 

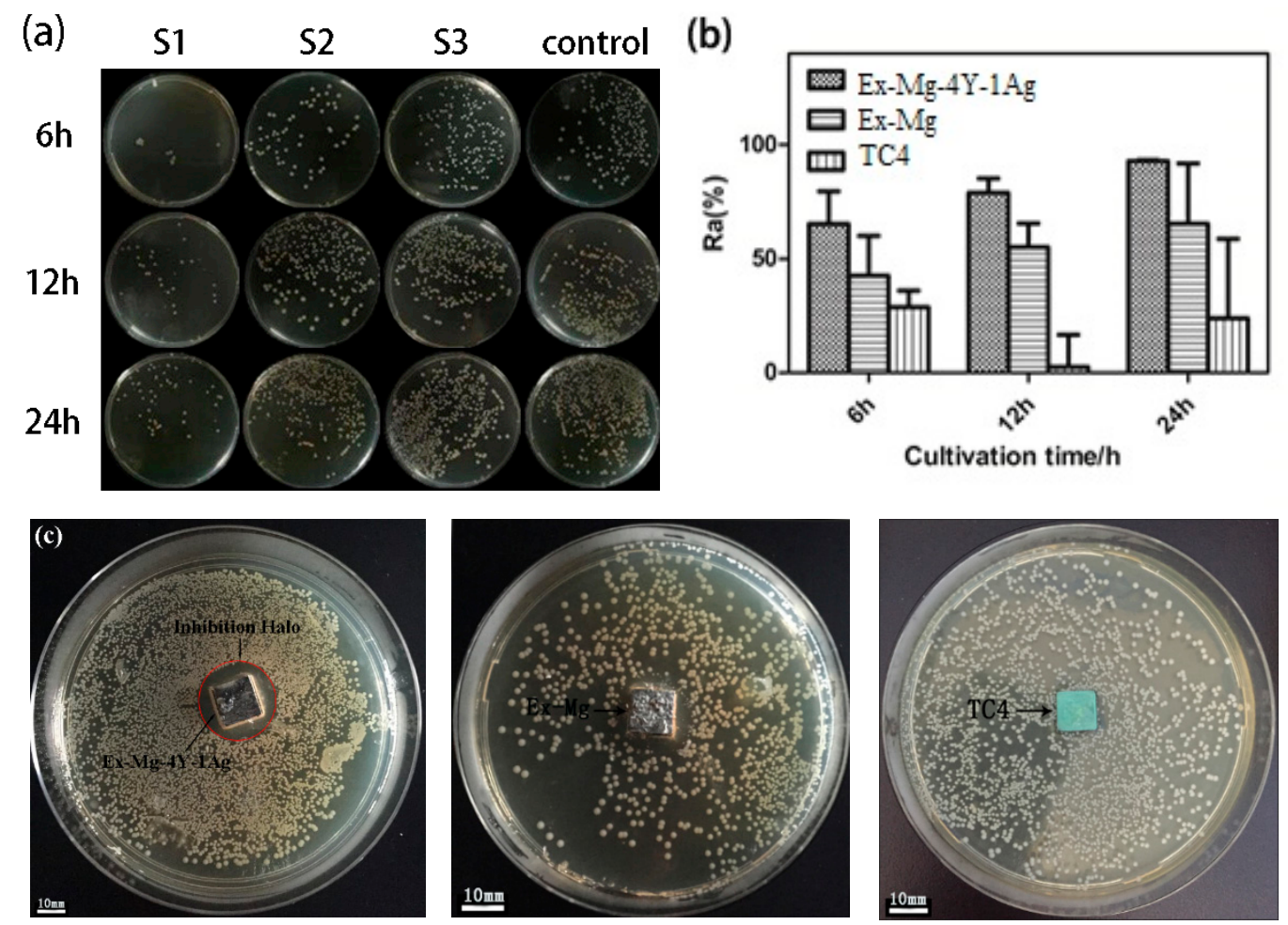

Figure 6. Results of bacteria counting and inhibition halo testing: (a) optical images of bacteria of three groups at $6 \mathrm{~h}, 12 \mathrm{~h}$ and $24 \mathrm{~h}$, respectively; (b) graph of the antibacterial rate of three groups at $6 \mathrm{~h}, 12 \mathrm{~h}$ and $24 \mathrm{~h}$; and (c) optical images of inhibition halo test for Ex-Mg-4Y-1Ag, Ex-Mg, TC4.

S1
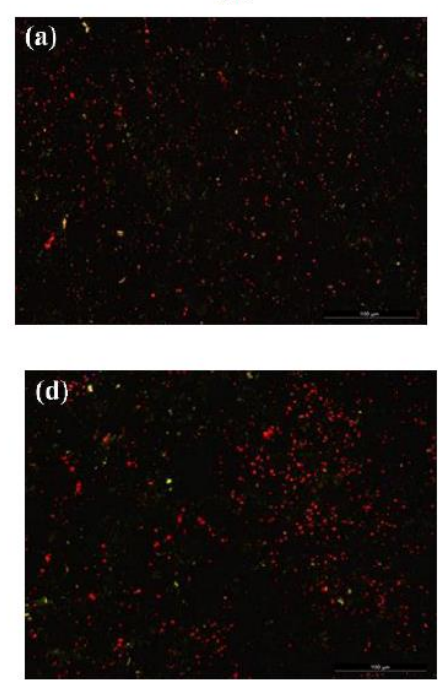

S2
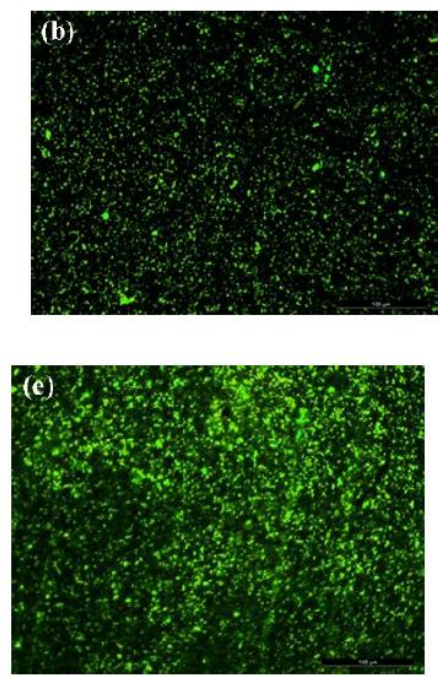

S3
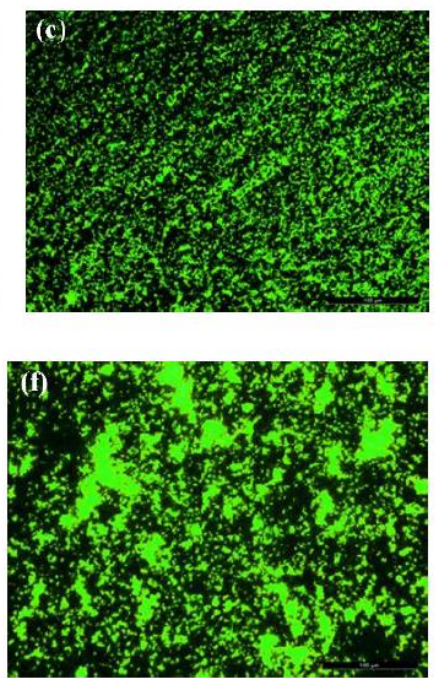

Figure 7. Fluorescence microscopy of Ex-Mg-4Y-1Ag, Ex-Mg, and TC4: (a-c) show S1, S2, S3 group cultured for $2 \mathrm{~h}$; (d-f) show S1, S2, S3 group cultured for $4 \mathrm{~h}$. 
Figure 8 shows the ion concentration variation of culture medium with samples in four weeks. The concentrations of $\mathrm{Ag}^{+}$in $\mathrm{S} 1$ reached their maximum values in approximately two weeks. It is found that the concentration of $\mathrm{Mg}^{2+}$ in S1 and S2 both remain a range from $18 \mathrm{mg} / \mathrm{L}$ to $30.84 \mathrm{mg} / \mathrm{L}$. The concentration of $\mathrm{Y}^{3+}$ was much smaller than the concentration of $\mathrm{Ag}^{+}$over the entire course of the experiment for the reason that the undissolved $\mathrm{Y}_{2} \mathrm{O}_{3}$ is the main product from the yttrium $[15,23]$. Moreover, there was no $\mathrm{Y}^{3+}$ ion could be detected in week 2, because all of $\mathrm{Y}$ element formed insoluble $\mathrm{Y}_{2} \mathrm{O}_{3}$ or $\mathrm{Y}(\mathrm{OH})_{3}$ corrosion product layer. Such a phenomenon indicates that the $\mathrm{Ag}^{+}$ion that was biodegraded from Ex-Mg-4Y-1Ag can inhibit bacteria proliferation for Ex-Mg-4Y-1Ag and Ex-Mg showing the close $\mathrm{pH}$ and concentration of $\mathrm{Mg}^{2+}$.
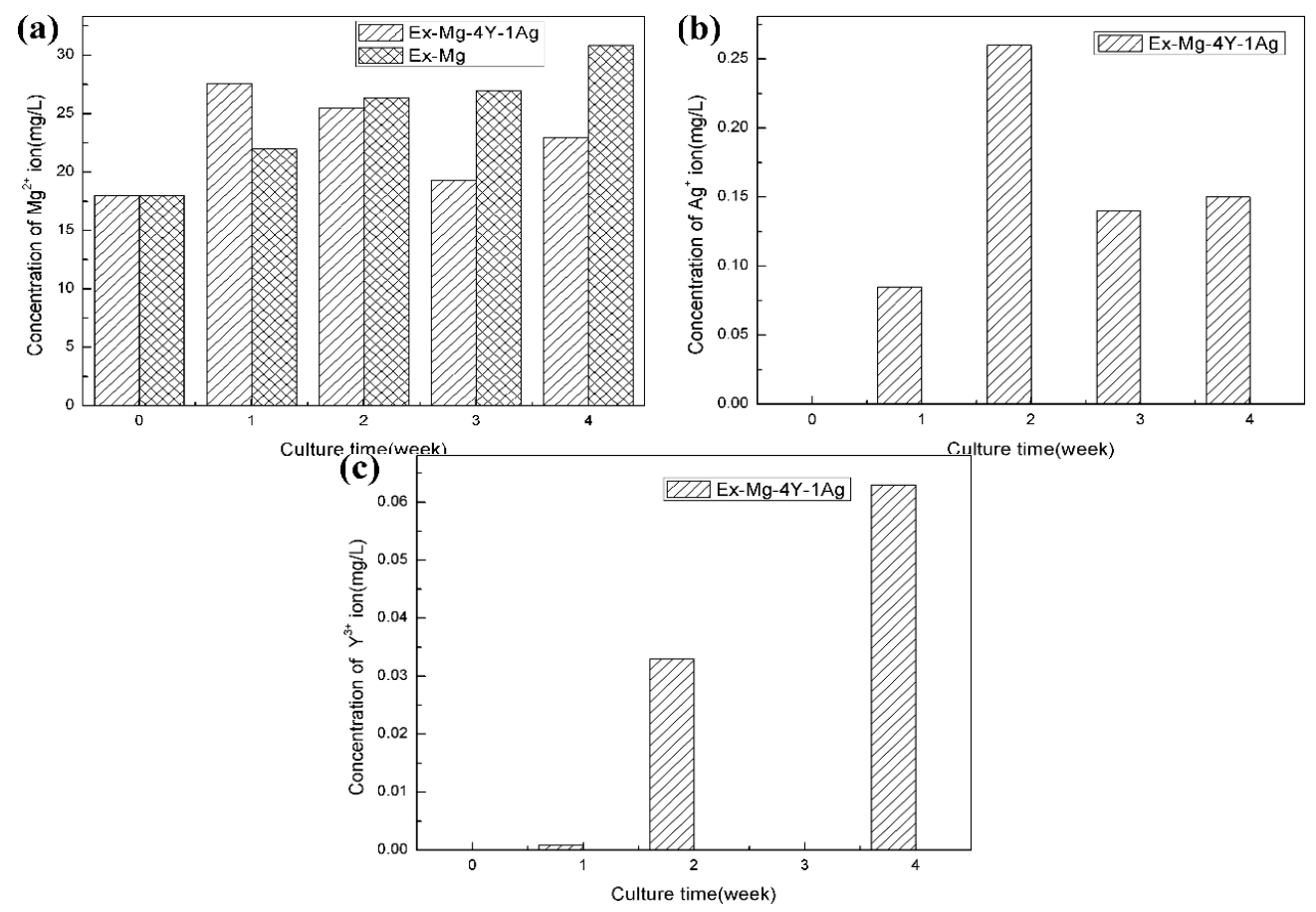

Figure 8. $\mathrm{Mg}^{2+}, \mathrm{Ag}^{+}, \mathrm{Y}^{3+}$ ion concentration variation of culture medium with Ex-Mg-4Y-1Ag and Ex-Mg: (a) $\mathrm{Mg}^{2+} ;$ (b) $\mathrm{Ag}^{+} ;$(c) $\mathrm{Y}^{3+}$.

\subsection{Cytotoxicity Test}

The cell toxicity experiment indicated that Optical value (OD) of the L-929 cells incubated in different concentration extracts of the Ex-Mg-4Y-1Ag alloy (Figure 9a), which can infer the cell relative growth rate (RGR) was between $83.0 \%$ and $99.4 \%$ in different dilution of extracting fluid and different time points. Thus, the RGR was calculated as following formula: RGR $(\%)=$ ODtest/ODnegative $\times 100 \%$. Based on ISO 10993-2009 standard, the cell toxicity scores were ranked in grade $0-1$, which means good biocompatibility in vitro. There were no significant difference between the $10 \%, 50 \%$ concentration extracting liquid groups and negative group (Figure $9 b-d)(p>0.05)$, while there was significant difference between $100 \%$ extracting liquid group (Figure 9e) and the positive group (Figure 9f) $(p<0.05)$. These results demonstrated that Ex-Mg-4Y-1Ag alloy showed no obvious cytotoxicity in vitro. 

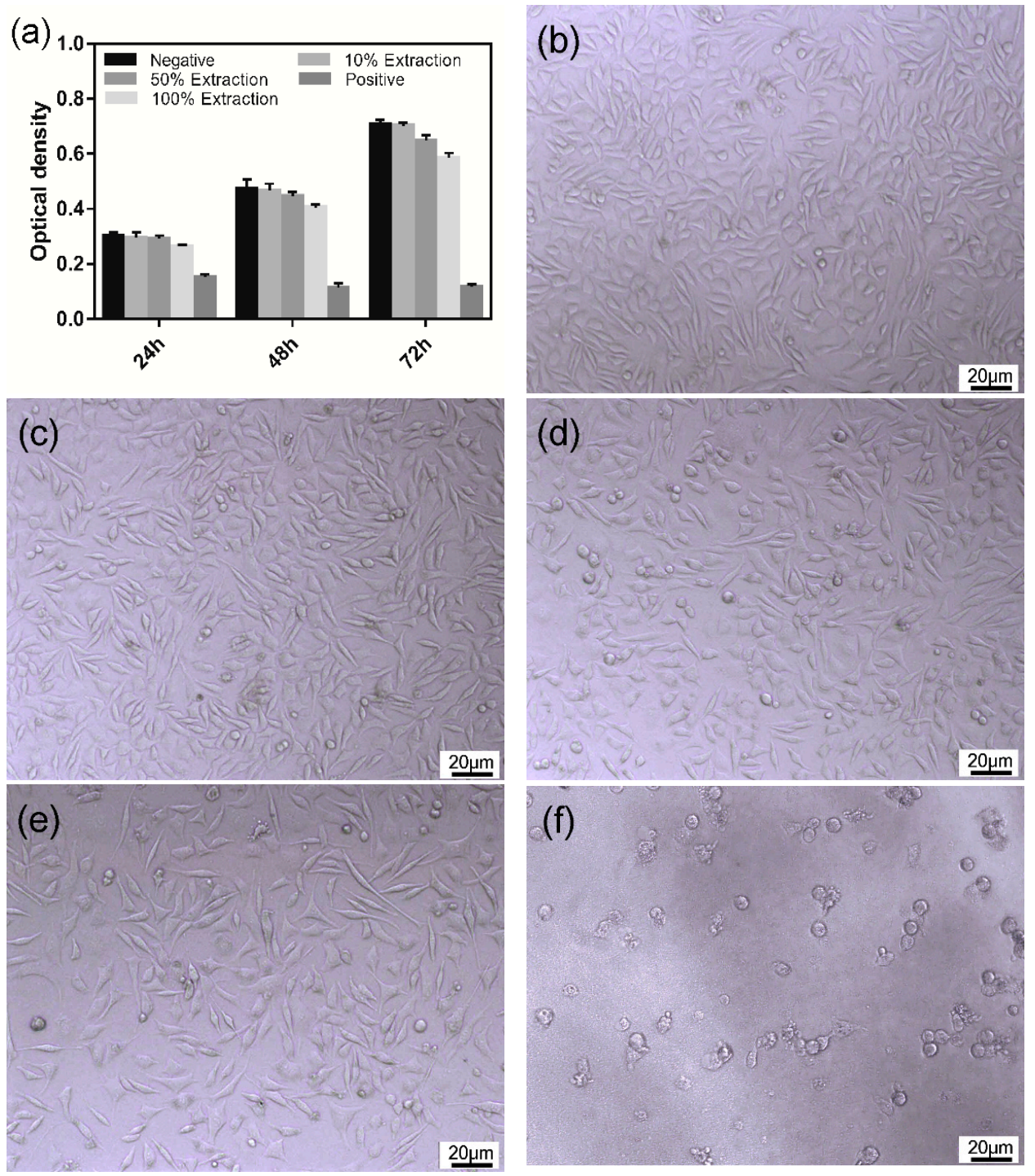

Figure 9. Cytotoxicity results of the Ex-Mg-4Y-1Ag alloy: (a) Optical value (OD) of L-929 cells cultured in different extracts. Optical images of L-929 cells that cultured in (b) negative control (complete medium); (c) 10\% extracting liquid; (d) 50\% extracting liquid; (e) 100\% extracting liquid; and (f) positive control $(0.5 \%$ phenol $)$ at $72 \mathrm{~h}$.

\section{Discussion}

The Ex-Mg-4Y-1Ag alloy exhibited good in mechanical, anticorrosion and antimicrobial properties because of their alloying and processing. The mechanical and in vitro corrosion tests demonstrated that the Mg-4Y-1Ag alloy which was processed by hot-extrusion showed better tensile strength, compressive strength and elongation than pure $\mathrm{Mg}$, and maintained good anticorrosion property. And it was selected for the analysis of antimicrobial property compared with Mg and TC4. Finally, the cytotoxicity results of the Ex-Mg-4Y-1Ag alloy illustrated no toxicity to L929 cells.

Hot-extrusion has been reported to be an efficient grain refiner for $\mathrm{Mg}$ alloys. In this work, the SEM and optical images confirmed that hot-extrusion in the Mg-4Y-1Ag alloy led to the refinement of the grain size. The mechanical properties including UTS and elongation of Mg-4Y-1Ag alloy were promoted for refined grains through hot-extrusion. Thus, hot-extrusion could get more refined grain and eliminate the second dendrites in grain. After hot-extrusion, the white second phases in as-extruded alloys decreased and distributed in homogeneous dispersion state. Hot-extrusion changed from localised corrosion in cast alloy to homogeneous corrosion properties in extruded alloy, 
which slowed down the corrosion of Mg-4Y-1Ag [24]. Thus, Ex-Mg-4Y-1Ag showed much better anticorrosion property than $\mathrm{C}-\mathrm{Mg}-4 \mathrm{Y}-1 \mathrm{Ag}$.

In vitro corrosion experiments are effective way to estimate degradable behaviour of the $\mathrm{Mg}$-based biomaterials in SBF solution. The $\mathrm{pH}$ value and hydrogen evolution variation in Figure 3 illustrated a phenomenon that degradation of $\mathrm{Mg}$ was slower than that of Ex-Mg-4Y-1Ag at beginning of $72 \mathrm{~h}$, however, the corrosion of Ex-Mg-4Y-1Ag slowed down after immersion of $120 \mathrm{~h}$ in PBS. At the beginning of degradation in PBS solution, the corrosion products of $\mathrm{Mg}$ and $\mathrm{Mg}$ alloy were hydrogen gas $\left(\mathrm{H}_{2}\right)$ and $\mathrm{Mg}(\mathrm{OH})_{2}$ according to Equation (1) [25].

$$
\begin{gathered}
\mathrm{Mg} \rightarrow \mathrm{Mg}^{2+}+2 \mathrm{e}^{-}(\text {anodic reaction }) \\
2 \mathrm{H}_{2} \mathrm{O}+2 \mathrm{e}^{-} \rightarrow \mathrm{H}_{2}+2 \mathrm{OH}^{-}(\text {cathodic reaction) } \\
\mathrm{Mg}+2 \mathrm{H}_{2} \mathrm{O} \rightarrow \mathrm{H}_{2}+\mathrm{Mg}(\mathrm{OH})_{2} \text { (overall reaction) }
\end{gathered}
$$

The corrosion rate (CR) was calculated by the equation below [26]:

$$
\text { Corrosion Rate }=95 \frac{\Delta \mathrm{V}}{\mathrm{S} \times \mathrm{T} \times \rho}
$$

where $\Delta \mathrm{V}(\mathrm{mL})$ is the $\mathrm{H}_{2}$ evolution of magnesium alloys, $\mathrm{S}\left(\mathrm{cm}^{2}\right)$ is the surface area exposed in Ringer's, $\mathrm{T}(\mathrm{h})$ is the immersion time and $\rho\left(\mathrm{g} / \mathrm{cm}^{3}\right)$ is the density of magnesium alloys.

The average corrosion rate of Mg and Ex-Mg-4Y-1Ag after $72 \mathrm{~h}, 120 \mathrm{~h}$, and $300 \mathrm{~h}$ of immersion was calculated in Table 4.

Table 4. The average corrosion rate of Mg and Ex-Mg-4Y-1Ag after $72 \mathrm{~h}, 120 \mathrm{~h}$, and $300 \mathrm{~h}$ of immersion.

\begin{tabular}{ccccc}
\hline CR (mm/year) Materials & $\mathbf{7 2} \mathbf{h}$ & $\mathbf{1 2 0} \mathbf{h}$ & $\mathbf{3 0 0} \mathbf{h}$ & $\mathbf{1}$ month \\
\hline $\mathrm{Mg}$ & 1.22 & 1.16 & 0.95 & 0.42 \\
Ex-Mg-4Y-1Ag & 2.36 & 1.52 & 0.86 & 0.35 \\
\hline
\end{tabular}

The surface of $\mathrm{Mg}$ alloy were covered by $\mathrm{Mg}(\mathrm{OH})_{2}$ layer, which could decrease the corrosion rate. However, the $\mathrm{Mg}(\mathrm{OH})_{2}$ layer was incompact and full of cracks as shown in Figure 4a. Then the PBS solution would touch the fresh surface through the cracks and continue the degradation process $[27,28]$. At the beginning of immersion, due to the potential of second phases in alloy ( $\varepsilon$ and $\mathrm{AgMg}_{4}$ ) is higher than the $\alpha \mathrm{Mg}$, so the Equation (1) reacted first. As shown in Table 4, the corrosion rate of Ex-Mg-4Y-1Ag alloy in PBS solution was bigger than that of $\mathrm{Mg}$ for the reason of galvanic corrosion accelerating reaction caused by the second phases in alloy. The chloride ions present in PBS solution transformed the protective $\mathrm{Mg}(\mathrm{OH})_{2}$ layer into the more resoluble $\mathrm{MgCl}_{2}$, according to Equation (3) [29]:

$$
\mathrm{Mg}(\mathrm{OH})_{2}+2 \mathrm{Cl}^{-} \rightarrow \mathrm{MgCl}_{2}+2 \mathrm{OH}^{-}
$$

At last, the dissolution and formation of an $\mathrm{Mg}(\mathrm{OH})_{2}$ layer produced a dynamic balance, so the dissoluted $\mathrm{Mg}^{2+}$ ion which was detected by ICP was part of degradable Mg element in $\mathrm{Mg}$ alloy. Therefore, the corrosion rate calculated by $\mathrm{Mg}^{2+}$ ion concentration in Figure 8 was smaller than that calculated by the $\mathrm{H}_{2}$ evolution.

After immersion of $120 \mathrm{~h}, \mathrm{Y}_{2} \mathrm{O}_{3}$ and $\mathrm{Y}(\mathrm{OH})_{3}$ were found in the corrosion products of Ex-Mg-4Y-1Ag from EDS (Figure 4). Finally, the corrosion of Ex-Mg-4Y-1Ag was slower than that 
of $\mathrm{Mg}$ after immersion of $300 \mathrm{~h}$. The following reactions Equation (4) [23] have occurred during the corrosion of Ex-Mg-4Y-1Ag alloy:

$$
\begin{gathered}
4 \mathrm{Y}+3 \mathrm{O}_{2} \rightarrow 2 \mathrm{Y}_{2} \mathrm{O}_{3} \\
\mathrm{Y}_{2} \mathrm{O}_{3}+3 \mathrm{H}_{2} \mathrm{O} \rightarrow 2 \mathrm{Y}(\mathrm{OH})_{3}
\end{gathered}
$$

Compared to the $\alpha \mathrm{Mg}=0.81$ (density of oxide film for $\mathrm{Mg}$ ), the value for $\mathrm{Y}$ is much bigger $(\alpha \mathrm{Y}$ $>1$ ), leading to a more compact mixture of $\mathrm{Mg}(\mathrm{OH})_{2}$ and $\mathrm{Y}_{2} \mathrm{O}_{3}$ or $\mathrm{Y}(\mathrm{OH})_{3}$ oxide film. The cracks on surface of Ex-Mg-4Y-1Ag were filled with $\mathrm{Y}_{2} \mathrm{O}_{3}$ or $\mathrm{Y}(\mathrm{OH})_{3}$, as shown in Figure 10. Therefore, the PBS solution could hardly touch the fresh surface, and the corrosion rate of Ex-Mg-4Y-1Ag decreased obviously. It has been reported that the beneficial effect of $Y$ is attributed to the eventual stabilization of the corrosion surface layer [15]. Liu et al. [23] found that for Mg-Y binary alloy, $\mathrm{Y}$ can incorporate in the surface film in the form of $\mathrm{Y}_{2} \mathrm{O}_{3}$ as confirmed with XPS and XRD, and can improve the corrosion resistance of the surface film.
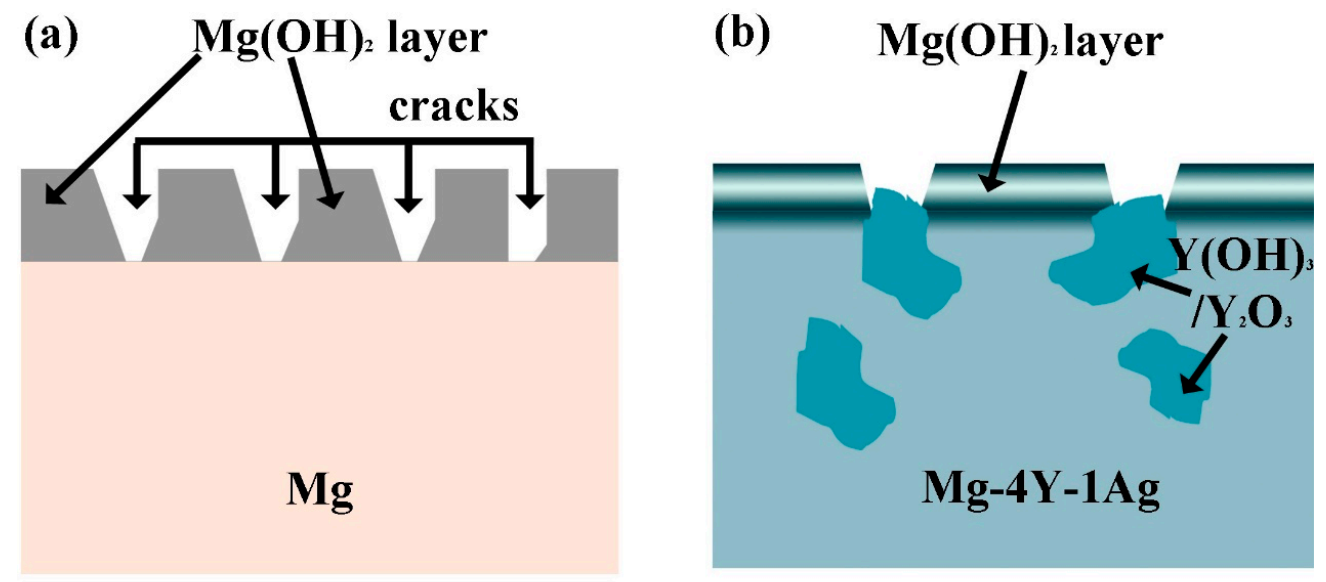

Figure 10. Surface corrosion layer formation on $\mathrm{Mg}$ and $\mathrm{Mg}-4 \mathrm{Y}-1 \mathrm{Ag}$ after exposure to SBF solution: (a) $\mathrm{Mg}$; (b) Mg-4Y-1Ag.

EIS plot and the equivalent circuit in Figure 5 illustrated the corrosion mechanism of $\mathrm{Mg}$ and $\mathrm{Mg}$ alloy in PBS solution. The diameter of high frequency capacitive loop is corresponding to the charge transfer resistance $R_{t}$ and could also reflect the value of electrochemical double layer. The $R_{t}$ of $\operatorname{Mg}\left(665 \Omega \cdot \mathrm{cm}^{2}\right)$ was a little bigger than that of Ex-Mg-4Y-1Ag $\left(430.9 \Omega \cdot \mathrm{cm}^{2}\right)$, which means $\mathrm{Mg}$ have better anticorrosion at the beginning of corrosion. The $\mathrm{R}_{\mathrm{f}}$ stood for the resistance of protective film, and it was mainly affected by compactness integrity of corrosion product layer [30]. It can be seen in Table 3 that $R_{\mathrm{f}}$ of Ex-Mg-4Y-1Ag $\left(521.6 \Omega \cdot \mathrm{cm}^{2}\right)$ was much bigger than that of $\mathrm{Mg}\left(2.404 \Omega \cdot \mathrm{cm}^{2}\right)$, which indicates the corrosion product layer of Ex-Mg-4Y-1Ag is much more compact than that of $\mathrm{Mg}$.

On one hand, the addition of Ag led to the improvement of the mechanical properties resulting from the grain refining effect of $\mathrm{Ag}$ [31]. On the other hand, the addition of $\mathrm{Ag}$ in $\mathrm{Mg}$ alloy showed good biocompatibility that Mg-Ag alloy exhibit no cytotoxicity of MG63 and RAW 264.7 cells, and antibacterial activity with the release of $\mathrm{Ag}^{+}$ion during the degradation [13]. As mentioned above in the antibacterial test, the order of antibacterial activity was Ex-Mg-4Y-1Ag $>\mathrm{Mg}>\mathrm{TC}$. The antibacterial activity of $\mathrm{Mg}$ derived from $\mathrm{OH}^{-}$ion which was its corrosion product. With the degradation of $\mathrm{Mg}$, both $\mathrm{OH}^{-}$ion and $\mathrm{pH}$ value of the local extract promote, and bacteria are hard to survive in alkaline environment. However, the $\mathrm{pH}$ value of PBS solution immersing with $\mathrm{Mg}$ and Ex-Mg-4Y-1Ag almost reach the same value $(\mathrm{pH}=9.1)$, Ex-Mg-4Y-1Ag exhibit better antibacterial activity than $\mathrm{Mg}$. Because the release of $\mathrm{Ag}^{+}$ion play more important role in antibacterial property. As shown in Figure 8, during the degradation of 4 weeks, the release of $\mathrm{Ag}^{+}$ion can be detected ranging from $0.085 \mathrm{mg} / \mathrm{L}-0.26 \mathrm{mg} / \mathrm{L}$, which ensure Ex-Mg-4Y-1Ag alloy implant keeping antibacterial activity during the whole degradation. 
The MTT assay was performed to continuously compare the proliferation of L929 cells cultured on different concentration of extracts of Mg-4Y-1Ag alloy according to ISO 10993-2009 standard. As shown in Figure 9a, an increasing trend of cell growth in a time dependent manner was observed in each extract group. And we found that high concentration (100\%) extract of Mg-4Y-1Ag alloy could inhibit cells proliferation $(p<0.05)$. However, no statistically significant difference was found in the proliferation rate of L929 cell among 10\% and 50\% concentration extracting liquid. These preliminary results showed that Ex-Mg-4Y-1Ag alloy exhibited good compatibility in vitro and further experimental studies are needed to be carried out to testify whether this alloy meets the requirements of biomedical materials.

\section{Conclusions}

(1) Hot-extrusion make Mg-4Y-1Ag alloys get smaller grain size and eliminate the second dendrites, which can greatly promote the corrosion resistance of $\mathrm{Mg}-4 \mathrm{Y}-1 \mathrm{Ag}$. The degradation rate of Ex-Mg-4Y-1Ag is close to Ex-Mg. The incorporation of oxidized $\mathrm{Y}$ in the surface film is identified as enhancing the degradation resistance of $\mathrm{Mg}-4 \mathrm{Y}-1 \mathrm{Ag}$ alloys.

(2) The Ex-Mg-4Y-1Ag alloys show acceptable strengths, elastic moduli and elongation. The densities and elastic moduli of the alloys match those of natural bone, and the strengths and elongation are larger than that of natural bone.

(3) Compared to Ex-Mg and TC4, the Ex-Mg-4Y-1Ag alloy possesses significant antibacterial effect toward S. aureus for its released $\mathrm{Ag}^{+}$ion and shows no cytotoxicity to L929 cells.

Author Contributions: For Y.D., X.X., Y.Y., and Y.Z. prepared the Mg-Y-Ag alloy samples and performed the microstructural characterization and the corrosion resistance under the supervision of K.Y., Y.T., H.L., and Z.L. performed antibacterial test and cell cytotoxicity under the supervision of Y.Z., Y.D. and H.L. revised manuscript. All authors discussed the results and approved the final manuscript.

Funding: This research was funded by the National Natural Science Foundation of China (No. 81874006), Fundamental Research Funds for the Central Universities of Central South University Project (2015zzts024), China, the Key Research and Development Program of Hunan Province project (2017GK2120), the Natural Science Foundation of Hunan Province of China (2018JJ2506), the Planned Science and Technology Project of Hunan Province, China, No. 2015SK20474, the 2015 ShanDong province project of outstanding subject talent group and the Natural Science Foundation of Shandong Province of China (ZR2017MEM005).

Conflicts of Interest: The authors declare no conflict of interest.

\section{References}

1. Witte, F. Reprint of the history of biodegradable magnesium implants: A review. Acta Biomater. 2015, 23, S28-S40. [CrossRef] [PubMed]

2. Zhang, Y.; Xu, J.; Ruan, Y.C.; Yu, M.K.; O’Laughlin, M.; Wise, H.; Chen, D.; Tian, L.; Shi, D.; Wang, J.; et al. Implant-derived magnesium induces local neuronal production of CGRP to improve bone-fracture healing in rats. Nat. Med. 2016, 22, 1160-1169. [CrossRef] [PubMed]

3. Yu, K.; Chen, L.; Zhao, J.; Li, S.; Dai, Y.; Huang, Q.; Yu, Z. In vitro corrosion behavior and in vivo biodegradation of biomedical $\beta$-Ca3(PO4)2/Mg-Zn composites. Acta Biomater. 2012, 8, 2845-2855. [CrossRef] [PubMed]

4. Song, G.L.; Song, S.Z. A possible biodegradable magnesium implant material. Adv. Eng. Mater. 2007, 9, 298-302. [CrossRef]

5. Mueller, W.D.; Nascimento, M.L.; de Mele, M. Critical discussion of the results from different corrosion studies of $\mathrm{Mg}$ and $\mathrm{Mg}$ alloys for biomaterial applications. Acta Biomater. 2010, 6, 1749-1755. [CrossRef] [PubMed]

6. Kirkland, N.T.; Birbilis, N.; Staiger, M.P. Assessing the corrosion of biodegradable magnesium implants: A critical review of current methodologies and their limitations. Acta Biomater. 2012, 8, 925-936. [CrossRef] [PubMed]

7. Witte, F.; Hort, N.; Vogt, C.; Cohen, S.; Kainer, K.U.; Willumeit, R.; Feyerabend, F. Degradable biomaterials based on magnesium corrosion. Curr. Opin. Solid State Mater. Sci. 2008, 12, 63-72. [CrossRef] 
8. Shi, Z.; Song, G.; Atrens, A. Corrosion resistance of anodised single-phase Mg alloys. Surf. Coat. Technol. 2006, 201, 492-503. [CrossRef]

9. Hedayati, R.; Ahmadi, S.M.; Lietaert, K.; Tã Mer, N.; Li, Y.; Amin, Y.S.; Zadpoor, A.A. Fatigue and quasi-static mechanical behavior of bio-degradable porous biomaterials based on magnesium alloys. J. Biomed. Mater. Res. A 2018, 106, 1798-1811. [CrossRef] [PubMed]

10. Peron, M.; Torgersen, J.; Berto, F. Mg and its alloys for biomedical applications: Exploring corrosion and its interplay with mechanical failure. Metals 2017, 7, 252. [CrossRef]

11. Du, H.; Wei, Z.; Liu, X.; Zhang, E. Effects of Zn on the microstructure, mechanical property and bio-corrosion property of Mg-3Ca alloys for biomedical application. Mater. Chem. Phys. 2011, 125, 568-575. [CrossRef]

12. Tie, D.; Feyerabend, F.; Hort, N.; Hoeche, D.; Kainer, K.U.; Willumeit, R.; Mueller, W.D. In vitro mechanical and corrosion properties of biodegradable Mg-Ag alloys. Mater. Corros. 2014, 65, 569-576. [CrossRef]

13. Tie, D.; Feyerabend, F.; Müller, W.; Schade, R.; Liefeith, K.; Kainer, K.U.; Willumeit, R. Antibacterial biodegradable Mg-Ag alloys. Eur. Cell. Mater. 2013, 25, 284-298. [CrossRef] [PubMed]

14. Feyerabend, F.; Fischer, J.; Holtz, J.; Witte, F.; Willumeit, R.; Drucker, H.; Vogt, C.; Hort, N. Evaluation of short-term effects of rare earth and other elements used in magnesium alloys on primary cells and cell lines. Acta Biomater. 2010, 6, 1834-1842. [CrossRef] [PubMed]

15. He, W.; Zhang, E.; Yang, K. Effect of Y on the bio-corrosion behavior of extruded Mg-Zn-Mn alloy in Hank's solution. Mater. Sci. Eng. C 2010, 30, 167-174. [CrossRef]

16. Nayak, S.; Bhushan, B.; Jayaganthan, R.; Gopinath, P.; Agarwal, R.D.; Lahiri, D. Strengthening of Mg based alloy through grain refinement for orthopaedic application. J. Mech. Behav. Biomed. 2016, 59, 57. [CrossRef] [PubMed]

17. Yan, Y.; Kang, Y.; Li, D.; Yu, K.; Xiao, T.; Deng, Y.; Dai, H.; Dai, Y.; Xiong, H.; Fang, H. Improvement of the mechanical properties and corrosion resistance of biodegradable $\beta$-Ca3(PO4)2/Mg-Zn composites prepared by powder metallurgy: The adding $\beta-\mathrm{Ca} 3(\mathrm{PO} 4) 2$, hot extrusion and aging treatment. Mater. Sci. Eng. C Mater. Biol. Appl. 2017, 74, 582. [CrossRef] [PubMed]

18. Vlček, M.; Lukáč, F.; Kudrnová, H.; Smola, B.; Stulíková, I.; Luczak, M.; Szakács, G.; Hort, N.; Willumeit-Römer, R. Microhardness and In Vitro Corrosion of Heat-Treated Mg-Y-Ag Biodegradable Alloy. Materials 2017, 10, 55. [CrossRef] [PubMed]

19. Song, G.; Atrens, A.; Stjohn, D. An Hydrogen Evolution Method for the Estimation of the Corrosion Rate of Magnesium Alloys; John Wiley \& Sons, Inc.: Hoboken, NJ, USA, 2013; pp. 255-262.

20. Peng, Q.; Huang, Y.; Zhou, L.; Hort, N.; Kainer, K.U. Preparation and properties of high purity Mg-Y biomaterials. Biomaterials 2010, 31, 398-403. [CrossRef] [PubMed]

21. Chen, Y.; Xu, Z.; Smith, C.; Sankar, J. Recent advances on the development of magnesium alloys for biodegradable implants. Acta Biomater. 2014, 10, 4561-4573. [CrossRef] [PubMed]

22. Pereda, M.D.; Alonso, C.; Burgos-Asperilla, L.; Del Valle, J.A.; Ruano, O.A.; Perez, P.; Ma, F.N.L.D. Corrosion inhibition of powder metallurgy $\mathrm{Mg}$ by fluoride treatments. Acta Biomater. 2010, 6, 1772-1782. [CrossRef] [PubMed]

23. Liu, M.; Schmutz, P.; Uggowitzer, P.J.; Song, G.; Atrens, A. The influence of yttrium (Y) on the corrosion of Mg-Y binary alloys. Corros. Sci. 2010, 52, 3687-3701. [CrossRef]

24. Zhou, W.; Shan, D.; Han, E.H.; Ke, W. Structure and formation mechanism of phosphate conversion coating on die-cast AZ91D magnesium alloy. Corros. Sci. 2008, 50, 329-337. [CrossRef]

25. Lebouil, S.; Duboin, A.; Monti, F.; Tabeling, P.; Volovitch, P.; Ogle, K. A novel approach to on line measurement of gas evolution kinetics: Application to the negative difference effect of $\mathrm{Mg}$ in chloride solution. Electrochim. Acta 2014, 124, 176-182. [CrossRef]

26. Witte, F.; Fischer, J.; Nellesen, J.; Crostack, H.A.; Kaese, V.; Pisch, A.; Beckmann, F.; Windhagen, H. In vitro and in vivo corrosion measurements of magnesium alloys. Biomaterials 2006, 27, 1013-1018. [CrossRef] [PubMed]

27. Abidin, N.I.Z.; Martin, D.; Atrens, A. Corrosion of high purity Mg, AZ91, ZE41 and Mg2Zn0.2Mn in Hank's solution at room temperature. Corros. Sci. 2011, 53, 862-872. [CrossRef]

28. Peng, Q.; Fu, H.; Pang, J.; Zhang, J.; Xiao, W. Preparation, mechanical and degradation properties of Mg-Y-based microwire. J. Mech. Behav. Biomed. 2014, 29, 375-384. [CrossRef] [PubMed] 
29. Ye, X.; Chen, M.; Yang, M.; Wei, J.; Liu, D. In vitro corrosion resistance and cytocompatibility of nano-hydroxyapatite reinforced Mg-Zn-Zr composites. J. Mater. Sci. Mater. Med. 2010, 21, 1321. [CrossRef] [PubMed]

30. Zhang, Y.; Yan, C.; Wang, F.; Li, W. Electrochemical behavior of anodized Mg alloy AZ91D in chloride containing aqueous solution. Corros. Sci. 2005, 47, 2816-2831. [CrossRef]

31. Fu, H.M.; Qiu, D.; Zhang, M.X.; Wang, H.; Kelly, P.M.; Taylor, J.A. The development of a new grain refiner for magnesium alloys using the edge-to-edge model. J. Alloys Compd. 2008, 456, 390-394. [CrossRef]

(C) 2018 by the authors. Licensee MDPI, Basel, Switzerland. This article is an open access article distributed under the terms and conditions of the Creative Commons Attribution (CC BY) license (http:// creativecommons.org/licenses/by/4.0/). 